\title{
Dose-response association between physical activity and sedentary time categories on ageing biomarkers
}

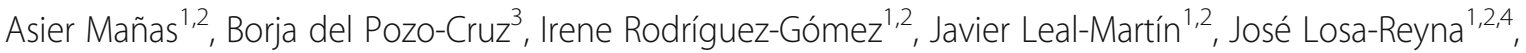
Leocadio Rodríguez-Mañas ${ }^{2,5}$, Francisco J. García-García ${ }^{2,4}$ and Ignacio Ara ${ }^{1,2^{*}}$ (1)

\begin{abstract}
Background: Physical activity and sedentary behaviour have been suggested to independently affect a number of health outcomes. To what extent different combinations of physical activity and sedentary behaviour may influence physical function and frailty outcomes in older adults is unknown. The aim of this study was to examine the combination of mutually exclusive categories of accelerometer-measured physical activity and sedentary time on physical function and frailty in older adults.

Methods: 771 older adults (54\% women; $76.8 \pm 4.9$ years) from the Toledo Study for Healthy Aging participated in this cross-sectional study. Physical activity and sedentary time were measured by accelerometry. Physically active was defined as meeting current aerobic guidelines for older adults proposed by the World Health Organization. Low sedentary was defined as residing in the lowest quartile of the light physical activity-to-sedentary time ratio. Participants were then classified into one of four mutually exclusive movement patterns: (1) 'physically active \& low sedentary', (2) 'physically active \& high sedentary', (3) 'physically inactive \& low sedentary', and (4) 'physically inactive \& high sedentary'. The Short Physical Performance Battery was used to measure physical function and frailty was assessed using the Frailty Trait Scale.

Results: 'Physically active \& low sedentary' and 'physically active \& high sedentary' individuals had significantly higher levels of physical function ( $\beta=1.73$ and $\beta=1.30$ respectively; all $p<0.001$ ) and lower frailty $(\beta=-13.96$ and $\beta=-8.71$ respectively; all $p<0.001$ ) compared to 'physically inactive \& high sedentary' participants. Likewise, 'physically inactive \& low sedentary' group had significantly lower frailty $(\beta=-2.50 ; p=0.05)$, but significance was not reached for physical function.

Conclusions: We found a dose-response association of the different movement patterns analysed in this study with physical function and frailty. Meeting the physical activity guidelines was associated with the most beneficial physical function and frailty profiles in our sample. Among inactive people, more light intensity relative to sedentary time was associated with better frailty status. These results point out to the possibility of stepwise interventions (i.e. targeting less strenuous activities) to promote successful aging, particularly in inactive older adults.
\end{abstract}

Keywords: Exercise, Ageing, Functioning and disability, Health behaviour, Lifestyle

\footnotetext{
* Correspondence: ignacio.ara@uclm.es

${ }^{1}$ GENUD Toledo Research Group, Universidad de Castilla-La Mancha, Avda.

Carlos III s/n, 45071 Toledo, Spain

${ }^{2}$ CIBER of Frailty and Healthy Aging (CIBERFES), Madrid, Spain

Full list of author information is available at the end of the article
}

(c) The Author(s). 2019 Open Access This article is distributed under the terms of the Creative Commons Attribution 4.0 International License (http://creativecommons.org/licenses/by/4.0/), which permits unrestricted use, distribution, and reproduction in any medium, provided you give appropriate credit to the original author(s) and the source, provide a link to the Creative Commons license, and indicate if changes were made. The Creative Commons Public Domain Dedication waiver (http://creativecommons.org/publicdomain/zero/1.0/) applies to the data made available in this article, unless otherwise stated. 


\section{Background}

There is compiling evidence showing the benefits of regular physical activity to improve physical functioning and reduce frailty among the elderly $[1,2]$. Consequently, physical activity, particularly more strenuous activity is now routinely recommended in the clinical management of frailty [2,3]. Blodgett et al. [4] and Manas et al. [5] have shown that moderate-to-vigorous physical activity (MVPA) is inversely associated with frailty and adverse health outcomes in middle-age ( $\geq 50$ years) and older adults ( $\geq 65$ years), respectively. However, few older adults meet the physical activity recommendations (i.e., $150 \mathrm{~min}$ of moderate intensity aerobic activity, $75 \mathrm{~min}$ of vigorous intensity aerobic activity, or an equivalent combination, in 10-min bouts [6]). In fact, previous research has found that older adults spend between 8 and $12 \mathrm{~h}$ of their waking day sedentary [7]. Sedentary behaviours, such as TV viewing, motorized transport, or leisure-time sitting, have been shown to contribute to adverse health outcomes in older people, including lower levels of physical functioning and higher levels of frailty $[4,8-10]$.

Nevertheless, we are far from a complete understanding of the inter-relationships between MVPA and sedentary behaviours and the role they may play on preserving physical function and reducing frailty levels among older adults. Several studies have shown that some people can meet the physical activity recommendations and yet display high levels of sedentary behaviours. The reverse could also be true. Thus, different combinations of behaviours (i.e. 'physically active and low sedentary', 'physically active and high sedentary', 'physically inactive and low sedentary', and 'physically inactive and high sedentary') are plausible during waking times. Potentially, these distinct combinations of behaviours may lead to a gradient of health consequences [11, 12]. For example, Bakrania et al. [11] found that physically active adults, even those who spent much of their time on sedentary behaviours, had better cardiometabolic health than those who were inactive. It was also suggested that those individuals with lower sedentary status in the absence of meeting the physical activity recommendations had better cardiometabolic health profile compared to those with higher sedentary status and that were physically inactive (i.e. did not meet the physical activity guidelines). This dose-response pattern has also been confirmed for biological markers and mortality in previous studies [12, 13].

Appreciation of potential physical function and frailty consequences that different combinations of mutually exclusive waking behaviours may have among older adults will be advantageous to target successful public health interventions. For instance, increasing light intensity physical activity could be a feasible approach to improve the physical functioning and reduce the level of frailty of older adults categorize as inactive and high sedentary. Further, if a dose-response exists between the different movement behaviour patterns and physical functioning/frailty in older individuals (i.e., if more active patterns of behaviour are associated with better health profiles), a gradual range of stepwise interventions can be proposed. For example, if someone is sitting in the lowest movement category (i.e., inactive, high sedentary), we could focus on an intervention that targets sedentary behaviour first to move that particular person from inactive, high sedentary to inactive, low sedentary. There are, however, no existing studies analysing the associations between mutually exclusive categories of physical activity and sedentary time with physical function and frailty in older adults. Therefore, the purpose of this study was to examine the combination of mutually exclusive categories of accelerometer-measured physical activity and sedentary time on physical function and frailty in a community-dwelling sample of older adults.

\section{Methods \\ Study design and participants}

The current study included a sample of 871 communitydwelling older adults (416 women) from wave 2 (2012 to 2014) and wave 3 (2015 to 2017) of the Toledo Study for Healthy Aging (TSHA) [14]. The methodology of the TSHA study has been described in detail elsewhere [5, 15]. Briefly, the TSHA is a population-based prospective cohort study originally conceived to explore the determinants and consequences of ageing and frailty in older adults from Toledo, Spain. All participants gave their written informed consent prior enrolment. All procedures were approved by the Clinical Research Ethics Committee of the Toledo Hospital and were conducted in accordance with the Declaration of Helsinki for human studies.

\section{Measurements \\ Frailty status}

Frailty was assessed by means of the Frailty Trait Scale (FTS) [16]. The FTS includes 7 domains calculated from 12 items including energy balance and nutrition, assessed using the body mass index, central obesity (waist circumference), unintentional weight loss and serum albumin levels; activity levels, assessed using the total score of the Physical Activity Scale for the Elderly [17]; the nervous system performance, evaluated based on was verbal fluency (estimated by asking the participants to give names of animals during one minute [18]) and balance (Romberg test [19]); the vascular system, measured by the brachial-ankle index done with Doppler ultrasound [20]; weakness, estimated with the grip strength in the dominant arm and the knee extension strength [14]; endurance, assessed by the chair stand 
test, which measures the number of times that a person stands up in $30 \mathrm{~s}$ [21]; and slowness, estimated by calculating the time to walk $3 \mathrm{~m}$ at a "normal pace" according to a standard protocol [19]. Scoring is detailed elsewhere [16]. The Total FTS score ranged from 0 (less frailty) to 100 (more frailty).

\section{Physical Function}

The Short Physical Performance Battery (SPPB) was used to assess physical function in this study [19]. Previous studies have shown that low scores on the SPPB have a high predictive value for a wide range of health consequences comprising disability [22], hospitalization [23], and death [24].

The SPPB measures gait speed (8- $\mathrm{ft}$ walk), standing balance, and lower extremity strength and endurance (chair rise task). A maximum of 4 points each for the balance, chair stand, and gait speed tests may be awarded, for a score between 0 and 12 (best), in which only integers are allowed [19].

\section{Physical activity and sedentary time assessment}

Physical activity and sedentary time were assessed via accelerometry (ActiTrainer and ActiGraph wGT3X-BT; ActiGraph, LLC, Pensacola, FL). Participants were instructed to wear an accelerometer on the left hip during waking hours for 7 consecutive days and to remove the accelerometer only before going to bed or for water activities [25]. A valid day was defined as having $\geq 480$ $\min (\geq 8 \mathrm{~h}$ ) of monitor wear, and the study included the results from participants with at least four valid days $[26,27]$. Accelerometer cut-points for sedentary time were $0-99 \mathrm{cpm}, 100-1951 \mathrm{cpm}$ for light physical activity, $1952-5724 \mathrm{cpm}$ for moderate physical activity, and $\geq 5725 \mathrm{cpm}$ for vigorous physical activity based on previously established cut-points [28]. These cut-off values have been used in previous analyses from the TSHA $[5,15]$. In addition, moderate physical activity, vigorous physical activity and MVPA time accumulated in bouts of $\geq 10 \mathrm{~min}$, allowing for a two-minute exception in the intensity threshold, were also derived. The total minutes in each intensity band were averaged over the number of valid days to estimate the mean time spent in each activity band.

\section{Physical activity and sedentary time categories determination}

We followed the methods outlined in Bakrania et al. [11] to classify participants in this study into 4 mutually exclusive behavioural categories according to their levels of physical activity and sedentary behaviour. Based on Bakrania et al. [11], and other studies [12, 29], the light physical activity-to-sedentary time ratio was used to classify participants in this study as low sedentary if they resided in the first quartile. Given that most of our sample was expected to be sedentary $[7,15]$, the remaining participants (i.e. those in quartiles 2 , 3, and 4 of light physical activity-to-sedentary time ratio) were classified as high sedentary. MVPA status was classified as 'physically active' or 'physically inactive' on the basis of whether or not participants met the WHO (World Health Organization) physical activity recommendations for older adults [30]. For this, at least one of these three premises had to be met: accumulate $150 \mathrm{~min}$ of moderate physical activity per week over periods of at least 10 min; accumulate $75 \mathrm{~min}$ of vigorous physical activity per week over periods of at least $10 \mathrm{~min}$, or accumulate 150 min per week of an equivalent combination of MVPA over periods of at least $10 \mathrm{~min}$.

Based on previous studies [31], four groups of mutually exclusive movement patterns were created: [1] 'physically active and low sedentary', [2] 'physically active and high sedentary', [3] 'physically inactive and low sedentary', and [4] 'physically inactive and high sedentary'.

\section{Confounding variables}

Participants were asked about their age, sex and ethnicity. Other socio-demographic variables such as education, income, and marital status were also self-reported in face-to-face interviews as described elsewhere [15].

\section{Statistical analysis}

Analyses were performed using the statistical software SPSS version 24.0 (IBM Corp., Armonk, NY). Participant characteristics of the full sample, stratified by each category, were tabulated. Mean (standard deviation) and frequency (percentage) were provided for continuous and categorical variables, respectively. Ternary plots with the three behaviours were generated to show the distribution of the sample compositions using $\mathrm{R}$ statistical system version 3.1.1. To test our hypothesis, a multiple linear regression analysis with the behavioural category as independent variable and frailty or physical function as dependent variable was fitted. Covariates in the model included: age, sex, education, marital status, and income. The 'physically inactive and high sedentary' category was selected as the reference category.

Also, the continuous association between time spent in sedentary activities as well as MVPA with the outcomes of interest in the study were explored via regression. The same set of covariates in addition to accelerometer wear time as well as both continuous MVPA time and sedentary status was used.

All analyses were two-sided where $p \leq 0.05$ was considered to be statistically significant. 


\section{Results}

Descriptive

Of the 871 eligible subjects, 100 participants had insufficient accelerometer wear time so 771 participants were finally included (Table 1).
The sample splits across the four different categories of movement as follows: [1] 'physically active and low sedentary': $n=38 ; 4.9 \%$, [2] 'physically active and high sedentary': $n=89 ; 11.5 \%$, [3] 'physically inactive and low sedentary': $n=154 ; 20.0 \%$, and [4] 'physically inactive

Table 1 Participant characteristics

\begin{tabular}{|c|c|c|c|c|c|}
\hline \multirow[t]{2}{*}{ Characteristics } & Sample & $\begin{array}{l}\text { 'Physically active } \\
\text { \& low sedentary' }\end{array}$ & $\begin{array}{l}\text { 'Physically active } \\
\text { \& high sedentary' }\end{array}$ & $\begin{array}{l}\text { 'Physically inactive } \\
\& \text { low sedentary' }\end{array}$ & $\begin{array}{l}\text { 'Physically inactive } \\
\text { \& high sedentary' }\end{array}$ \\
\hline & $N=771$ & $n=38 ; 4.9 \%$ & $n=89 ; 11.5 \%$ & $n=154 ; 20.0 \%$ & $n=490 ; 63.6 \%$ \\
\hline Age (years) $^{a}$ & $76.8(4.9)$ & $74.4(4.0)$ & $74.8(3.7)$ & $75.9(4.5)$ & $77.7(5.1)$ \\
\hline \multicolumn{6}{|l|}{$\operatorname{Sex}{ }^{b}$} \\
\hline Male & $355(46.0)$ & $23(60.5)$ & $62(69.7)$ & $50(32.5)$ & $220(44.9)$ \\
\hline Female & $416(54.0)$ & $15(39.5)$ & $27(30.3)$ & $104(67.5)$ & $270(55.1)$ \\
\hline \multicolumn{6}{|l|}{ Education b } \\
\hline None & $487(63.2)$ & $19(50.0)$ & $46(51.7)$ & $97(63.0)$ & $325(66.3)$ \\
\hline Primary school & $169(21.9)$ & $11(28.9)$ & $26(29.2)$ & $39(25.3)$ & $93(19.0)$ \\
\hline Secundary or more & $109(14.1)$ & $8(21.1)$ & $17(19.1)$ & $16(10.4)$ & 68 (13.9) \\
\hline Missing ${ }^{c}$ & $6(0.8)$ & $0(0.0)$ & $0(0.0)$ & $2(1.3)$ & $4(0.8)$ \\
\hline \multicolumn{6}{|l|}{ Income ${ }^{b}$} \\
\hline Low & $369(47.8)$ & $23(60.5)$ & $45(50.6)$ & $66(42.8)$ & $235(48.0)$ \\
\hline Medium & $299(38.8)$ & $11(29.0)$ & $33(37.1)$ & $75(48.7)$ & $180(36.7)$ \\
\hline High & $56(7.3)$ & $3(7.9)$ & $6(6.7)$ & $7(4.6)$ & $40(8.1)$ \\
\hline Missing ${ }^{c}$ & $47(6.1)$ & $1(2.6)$ & $5(5.6)$ & $6(3.9)$ & $35(7.1)$ \\
\hline \multicolumn{6}{|l|}{ Marital status ${ }^{b}$} \\
\hline Single & $42(5.4)$ & $2(5.3)$ & $1(1.1)$ & $9(5.8)$ & $30(6.1)$ \\
\hline Married & $541(70.2)$ & $28(73.7)$ & $73(82.0)$ & $112(72.7)$ & $328(66.9)$ \\
\hline Widowed & $171(22.2)$ & 7 (18.4) & $13(14.6)$ & $30(19.5)$ & $121(24.7)$ \\
\hline Divorced/Separated & $12(1.6)$ & $1(2.6)$ & $2(2.2)$ & $0(0.0)$ & $9(1.8)$ \\
\hline Missing ${ }^{c}$ & $5(0.6)$ & $0(0.0)$ & $0(0.0)$ & $3(1.9)$ & $2(2.4)$ \\
\hline Body mass index $\left(\mathrm{kg} / \mathrm{m}^{2}\right)^{a}$ & $30.3(4.8)$ & $26.9(3.8)$ & $28.8(3.6)$ & $30.2(4.4)$ & $30.8(5.0)$ \\
\hline Short physical performance battery (points) ${ }^{a}$ & $8.4(3.2)$ & $10.7(1.6)$ & $10.2(2.1)$ & $8.4(2.9)$ & $7.9(3.4)$ \\
\hline Missing ${ }^{c}$ & $6(0.8)$ & $0(0.0)$ & $0(0.0)$ & $1(0.6)$ & $5(1.0)$ \\
\hline Frailty trait scale (points) ${ }^{a}$ & $38(14.5)$ & $23.6(11.7)$ & $28.9(11.8)$ & $37.7(13.9)$ & $40.9(13.9)$ \\
\hline Missing ${ }^{c}$ & $22(2.9)$ & $0(0.0)$ & $0(0.0)$ & $4(2.6)$ & $18(3.7)$ \\
\hline Accelerometer wear time (min/valid day) ${ }^{a}$ & 786.0 (82.6) & $810.0(84.3)$ & $828.9(80.1)$ & $799.9(81.5)$ & 772.0 (79.6) \\
\hline Sedentary time (min/valid day) ${ }^{a}$ & $539.9(90.6)$ & $433.2(46.7)$ & $557.9(67.4)$ & $447.0(65.1)$ & $574.0(76.1)$ \\
\hline Light physical activity (min/valid day) ${ }^{a}$ & $226.8(86.2)$ & $311.6(50.1)$ & $211.9(44.0)$ & $337.1(58.4)$ & $188.2(64.5)$ \\
\hline $\begin{array}{l}\text { Moderate-to-vigorous physical activity } \\
\text { (min/valid day) }^{a}\end{array}$ & $19.4(23.8)$ & $65.2(22.0)$ & $59.1(23.8)$ & $15.8(13.8)$ & $9.8(12.1)$ \\
\hline $\begin{array}{l}\geq 10 \text {-min bouts of moderate-to-vigorous } \\
\text { physical activity (min/day) }\end{array}$ & $9.6(17.7)$ & $42.6(18.2)$ & $42.6(22.3)$ & $4.3(6.1)$ & $2.7(5.1)$ \\
\hline \multicolumn{6}{|l|}{ Meet WHO guidelines ${ }^{b}$} \\
\hline Yes & $127(16.5)$ & $38(100.0)$ & 89 (100.0) & $0(0.0)$ & $0(0.0)$ \\
\hline No & $644(83.5)$ & $0(0.0)$ & $0(0.0)$ & $154(100.0)$ & $490(100.0)$ \\
\hline Light physical activity-to-sedentary time ratio ${ }^{a}$ & $0.45(0.24)$ & $0.72(0.11)$ & $0.39(0.09)$ & $0.78(0.26)$ & $0.34(0.13)$ \\
\hline
\end{tabular}

${ }^{a}$ Continuous variable; Mean (Standard Deviation)

${ }^{\mathrm{b}}$ Categorical variable; $\mathrm{n}$ (Proportion (\%))

cMissing data; n (\%) 
and high sedentary': $n=490 ; 63.6 \%$. Ternary plots represent the time spent in each movement behaviour at a time for the different categories (Fig. 1).

Compared to 'physically inactive and high sedentary' participants, 'physically active and low sedentary' and 'physically active and high sedentary' individuals had significantly higher levels of physical functioning $(\beta=1.73$; confidence interval $[\mathrm{CI}]=0.77,2.68$; and $\beta=1.30 ; C I=0.63,1.98$; respectively; $p<0.001)$ and lower frailty trait $(\beta=-13.96 ; C I=-18.31,-9.62$; and $\beta=-8.71 ; C I=-11.77,-5.65$; respectively; $p<$ 0.001). Furthermore, 'physically inactive and low sedentary' group had significantly lower frailty score $(\beta=-2.50 ; \mathrm{CI}=-4.98,-0.03 ; p<0.05)$. However, differences on physical function between this two groups were not significant $(\beta=0.31 ; C I=-0.23$, $0.84 ; p=0.26)$ (Table 2).

Increased time spent in MVPA was significantly associated with higher levels of physical functioning $(p<0.001)$ and lower frailty trait $(p<0.001)$. Likewise, a higher light physical activity-to-sedentary time ratio was significantly associated with higher physical functioning score $(p=0.03)$ and lower frailty trait $(p=$ 0.008) (Table 3).

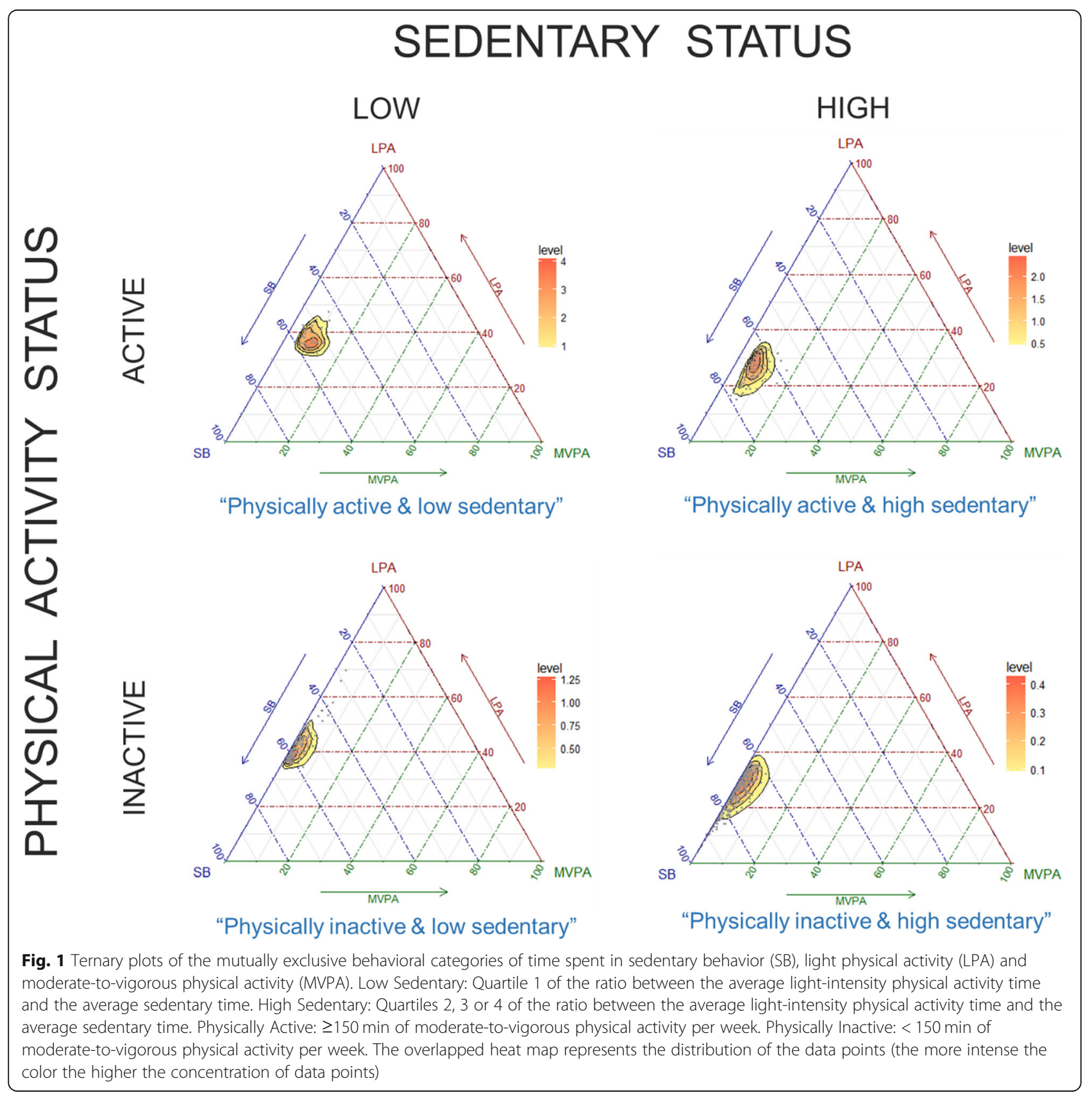


Table 2 Categorical associations with physical function and frailty (beta coefficients ( $95 \%$ Cls) and corresponding p-values)

\begin{tabular}{|c|c|c|c|c|c|c|c|}
\hline \multirow[t]{2}{*}{ Outcome } & \multicolumn{2}{|l|}{$\begin{array}{l}\text { 'Physically active } \\
\text { \& low sedentary' }\end{array}$} & \multicolumn{2}{|l|}{$\begin{array}{l}\text { 'Physically active } \\
\& \text { high sedentary' }\end{array}$} & \multicolumn{2}{|l|}{$\begin{array}{l}\text { 'Physically inactive } \\
\text { \& low sedentary' }\end{array}$} & \multirow[t]{2}{*}{$\begin{array}{l}\text { 'Physically inactive } \\
\text { \& high sedentary' }\end{array}$} \\
\hline & Beta $(95 \% \mathrm{Cl})$ & $p$-value & Beta $(95 \% \mathrm{Cl})$ & $p$-value & Beta $(95 \% \mathrm{Cl})$ & $p$-value & \\
\hline $\begin{array}{l}\text { Short Physical Performance } \\
\text { Battery }(n=765)\end{array}$ & $1.73(0.77,2.68)$ & $<0.001$ & $1.30(0.63,1.98)$ & $<0.001$ & $0.31(-0.31,0.84)$ & 0.263 & Reference \\
\hline Frailty Trait Scale $(n=749)$ & $-13.96(-18.31,-9.62)$ & $<0.001$ & $-8.71(-11.77,-5.65)$ & $<0.001$ & $-2.50(-4.98,-0.03)$ & 0.047 & Reference \\
\hline
\end{tabular}

Adjusted linear regression models were fitted for physical function and frailty outcomes. The models were controlled for: age, sex, education, income and marital status

Bold indicates statistical significance at $a=0.05$

\section{Discussion}

The way in which time packed in a given day remains relevant for a wide range of health outcomes [32]. Previous research has identified the cardiometabolic [11] and mortality outcomes [13] of different movement patterns in adults and older adults, respectively. This is the first study assessing the associations of mutually exclusive categories of accelerometer-derived physical activity and sedentary time with physical function and frailty in older adults. The main findings were that participants who engaged in $\geq 150 \mathrm{~min} /$ week of MVPA had more favourable physical function and frailty profiles than those classified in the other movement pattern groups, regardless of sedentary status. Our results also suggest that engaging in more light intensity relative to sedentary time may have a positive impact on physical function and frailty status on the studied population, even in those individuals already meeting the physical activity guidelines. This might provide alternative intervention strategies to improve physical function and prevent frailty, as light activities are more feasible than more strenuous activity, particularly among previously inactive individuals.

Previous research have demonstrated that MVPA is effective to prevent, delay or even reverse functional limitations and frailty among older adults [33]. The present study provides novel data indicating that older adults who meet recommended physical activity levels, regardless of time spent in light-intensity activities relative to sedentary activities, have better physical function levels and frailty status compared to older adults who do not meet the required physical activity levels. These results emphasize the importance of engaging in sufficient MVPA, which could buffer some of the negative consequences of sedentary behaviour in preserving the physical functionality and reduce frailty in this population group [34, 35]. A recent meta-analysis involving more than 1 million adults has shown that engaging in higher amounts of strenuous activity can eliminate the mortality risk associated with too much sitting reported elsewhere [36]. The association of more intense activity with fitness levels partially explains why meeting the physical activity recommendations may overcome the harmful effects of sedentary behaviours. Thus, cardiovascular fitness has been proposed as a plausible mechanism mediating the relationship between sedentary behaviour and cardiometabolic health in older adults [37]. More studies are required to elucidate the role of fitness in the relationship between MVPA, sedentary behaviour, physical functioning and frailty in older adults.

Contemporary experimental $[38,39]$ and observational $[40,41]$ evidence emphasizes the health-enhancing role of light-intensity activities. In a recent meta-analysis by Chastin et al. [42], light-intensity physical activity emerged as potentially relevant for cardiometabolic health and mortality in adults and older adults, in particular among impaired individuals. Our estimates suggest that increasing the time in light physical activity relative to sedentary time has a positive impact on frailty levels in those considered physically inactive. Other studies have suggested the potential benefits of replacing sedentary behaviour with light-intensity physical activity to reduce frailty in older adults with multiple diseases [5].

Table 3 Continuous associations with physical function and frailty (beta coefficients (95\% Cls) and corresponding p-values)

\begin{tabular}{|c|c|c|c|c|}
\hline \multirow[t]{2}{*}{ Outcome } & \multicolumn{2}{|c|}{ Moderate-to-vigorous physical activity time } & \multicolumn{2}{|c|}{ Light physical activity-to-sedentary behavior ratio } \\
\hline & Beta $(95 \% \mathrm{Cl})^{\text {a }}$ & $p$-value & Beta $(95 \% \mathrm{Cl})^{\mathrm{b}}$ & $p$-value \\
\hline $\begin{array}{l}\text { Short Physical Performance } \\
\text { Battery }(n=765)\end{array}$ & $0.03(0.02,0.04)$ & $<0.001$ & $0.96(0.09,1.82)$ & 0.030 \\
\hline Frailty Trait Scale $(n=749)$ & $-0.18(-0.22,-0.14)$ & $<0.001$ & $-5.39(-9.34,-1.44)$ & 0.008 \\
\hline
\end{tabular}

Adjusted linear regression models were fitted for physical function and frailty outcomes. The models were controlled for: age, sex, education, income, marital status, moderate-to-vigorous physical activity time, light physical activity-to-sedentary time ratio, and accelerometer wear-time.

Bold indicates statistical significance at $a=0.05$

${ }^{a}$ Beta coefficients represent a one minute increase in moderate-to-vigorous physical activity time per day

${ }^{\mathrm{b}}$ Beta coefficients represent a one unit increase in the light physical activity-to-sedentary behavior ratio 
It might be the case that in the more frail and functionally compromised individuals even small stimulus from light intensities can benefit their wider health [5]. Collectively, these findings are policy-relevant. Light-intensity physical activity is normally naturally embedded into the daily living of individuals (e.g. walking a dog, doing home chores or standing up while talking on the phone), therefore requiring no mental or physical effort or starting level to perform such activities, and thereby making lightintensity activities a pragmatic target for future public interventions to reduce frailty and improve physical function of older adults, particularly among those inactive (i.e. $83.5 \%$ in our sample) and that also depict very high levels of sedentary time (i.e. $63.6 \%$ in our sample) which might also be the most impaired individuals.

Interestingly, we identified the group meeting the physical activity guidelines (i.e. active) and showing higher levels of light intensity relative to sedentary time as the group with better frailty and physical function profile in our sample. Others have found similar results for cardiometabolic health [11] and mortality [13]. Recent epidemiologic evidence suggests that sitting time has deleterious cardiovascular and metabolic effects that are independent of whether or not adults meet the physical activity guidelines [31]. Our results suggest that engaging in more light-intensity activity relative to sedentary time beyond meeting the physical activity recommendations can provide with extra benefits in improving physical function and reducing frailty in older adults. Those individuals in our sample meeting the physical activity guidelines and engaging in more lightintensity activities extend their total volume of physical activity as supposed to those that meet the recommended amount of physical activity yet are sedentary, which could partially explain the extra benefit associated to that movement pattern [43]. Thus, promoting lightintensity activities could be a good approach to increase the total volume of physical activity and reduce sedentary time in those already meeting the physical activity guidelines, thereby enhancing their health, including increasing physical function and improving their frailty profile.

\section{Strengths and limitations}

The present study has several strengths. First, the study includes a relatively-large sample of community-dwelling older adults with advanced age. Although there is no current established gold standard to determine physical function and frailty in older adults, the short physical function battery has positioned as one of the most used tools to objectively evaluate functional performance among older adults [44]. Similarly, the Frailty Trait Scale has been suggested as a more sensitive scale for detecting changes in the individual's biological status than previously validated frailty instruments [16]. We also used accelerometer-measured procedures to assess physical activity and sedentary time.

Our study has also limitations. Firstly, the cut-off points used in the study to categorize the activity intensity of participants in the study can lead to a misclassification of both physical activity and sedentary time. However, the cut-off points used in this study are the most commonly reported in the literature for this age group [45], which make the results found here comparable with other investigations. Furthermore, ActiGraph devices are not able to discriminate between sitting and standing changes in the posture [46]. In order to obtain the activity status, bouts of at least $10 \mathrm{~min}$ were used, which may underestimate the time spent in MVPA. Nevertheless, further research is needed to consider the impact of the bout duration on frailty syndrome. Similar to what Bakrania et al. [11] reported, data could be overestimating the sedentary time [47], we therefore decided to use a more conservative approach for the extraction of sedentary status based on the behaviour of our population, an approach used in previous studies [11]. Loprinzi et al. defined low sedentary status as a positive light physical activity-to-sedentary time ratio [12]. If we had used the Loprinzi et al. [12] method, only $2.1 \%$ of our population would have been categorized as low sedentary status. This procedure used may have limitations and strengths. On the one hand, it is not influenced by the measurement of the accelerometer, but on the other hand, because is data-driven, may not be applicable to other populations. The use of this novel approach allows combining in mutually exclusive categories that best represent the different plausible combinations of physical activity and sedentary time within waking hours. Nonetheless, the cross-sectional nature of the research design used does not allow definitive conclusions to be drawn around the causal relationship between the outcomes of the study.

\section{Conclusions}

We observed that physically active older adults had better physical function and frailty profiles than those considered physically inactive, even in the presence of high sedentary time. Higher levels of light-intensity physical activity relative to sedentary time seems to provide additional benefits in both physical function and frailty outcomes among those meeting the physical activity guidelines. Lower sedentary levels were associated with decreased frailty in physically inactive participants. Altogether, our findings reinforce the idea of the healthenhancing benefits of meeting the current physical activity guidelines. Also, our results highlight the relevance of light-intensity physical activity for inactive older adults. If our results remain experimentally true, light intensity 
physical activity can be promoted as a middle step intervention among inactive individuals to achieve the recommended levels of physical activity and improve their health. We should move beyond observational studies and confirm our results in well-design longitudinal, experimental studies.

\section{Abbreviations}

FTS: Frailty Trait Scale; MVPA: Moderate-to-Vigorous Physical Activity; SPPB: Short Physical Performance Battery; TSHA: Toledo Study for Healthy Aging; WHO: World Health Organization

\section{Acknowledgements}

The authors would like to thank the cohort members, investigators, research associates, and team members.

\section{Authors' contributions}

AM: conceptualization, investigation, data curation, formal analysis, writing original draft preparation; BdPC, IRG, JLM \& JLR: investigation, data curation, writing/review \& editing; LRM, FJGG \& IA: funding acquisition, writing/review \& editing; AM, BdPC and IA performed the statistical analyses and are guarantors. All the authors read the draft, made contributions and approved the final manuscript

\section{Funding}

This work was supported by the Biomedical Research Networking Center on Frailty and Healthy Aging (CIBERFES) and FEDER funds from the European Union (CB16/10/00477) and (CB16/10/00456). It was further funded by grants from the Government of Castilla-La Mancha (PI2010/020; Institute of Health Sciences, Ministry of Health of Castilla-La Mancha, 03031-00), Spanish Government (Spanish Ministry of Economy, "Ministerio de Economía y Competitividad," Instituto de Salud Carlos III, PI10/01532, PI031558, PI11/01068), and by European Grants (Seventh Framework Programme: FRAILOMIC). Asier Mañas Bote and Irene Rodríguez Gómez received a PhD grant from the Universidad de Castilla-La Mancha "Contratos predoctorales para la formación de personal investigador en el marco del Plan Propio de I+D+i, cofinanciados por el Fondo Social Europeo" (2015/4062 and 2014/10340, respectively). These funding bodies played no role in the design of the study, collection, analysis, and interpretation of data or in writing the manuscript.

\section{Availability of data and materials}

There is an established infrastructure, including a website (http://http://www. ciberfes.es/) and a review committee, through which data requests are handled. The hospital reviews and determines the purposes for the data requests and what data can be released. Data requests can be sent to: Research and teaching unit, Virgen del Valle Hospital Ctra. Cobisa S/N, 45071 Toledo - Spain, info@estudiotoledo.com.

\section{Ethics approval and consent to participate}

All participants volunteered and signed an informed consent. All the objectives, physical tests, and aspects related to the methodology were explained orally and in writing. Data confidentiality and anonymity were guaranteed in all phases of the study following the 2009 law regulating personal data protection. The study was approved by the appropriate Ethics Committee (The Clinical Research Ethics Committee of the Toledo Hospital June 2012).

\section{Consent for publication}

Not applicable.

\section{Competing interests}

The authors declare that they have no competing interests.

\section{Author details}

${ }^{1}$ GENUD Toledo Research Group, Universidad de Castilla-La Mancha, Avda. Carlos III s/n, 45071 Toledo, Spain. ${ }^{2}$ CIBER of Frailty and Healthy Aging (CIBERFES), Madrid, Spain. ${ }^{3}$ Motivation and Behaviour Research Program, Institute for Positive Psychology and Education, Faculty of Health Sciences, Australian Catholic University, Sydney, Australia. ${ }^{4}$ Geriatric Department,
Hospital Virgen del Valle, Toledo, Spain. ${ }^{5}$ Geriatric Department, Hospital Universitario de Getafe, Getafe, Spain.

Received: 22 January 2019 Accepted: 16 September 2019 Published online: 15 October 2019

\section{References}

1. Pahor M, Guralnik JM, Ambrosius WT, Blair S, Bonds DE, Church TS, et al. Effect of structured physical activity on prevention of major mobility disability in older adults: the LIFE study randomized clinical trial. Jama. 2014; 311(23):2387-96.

2. Puts MTE, Toubasi S, Andrew MK, Ashe MC, Ploeg J, Atkinson E, et al. Interventions to prevent or reduce the level of frailty in communitydwelling older adults: a scoping review of the literature and international policies. Age Ageing. 2017;46(3):383-92.

3. Daly RM, Ahlborg HG, Ringsberg K, Gardsell P, Sernbo I, Karlsson MK. Association between changes in habitual physical activity and changes in bone density, muscle strength, and functional performance in elderly men and women. J Am Geriatr Soc. 2008;56(12):2252-60.

4. Blodgett J, Theou O, Kirkland S, Andreou P, Rockwood K. The association between sedentary behaviour, moderate-vigorous physical activity and frailty in NHANES cohorts. Maturitas. 2015;80(2):187-91.

5. Manas A, Del Pozo-Cruz B, Guadalupe-Grau A, Marin-Puyalto J, Alfaro-Acha A, Rodriguez-Manas L, et al. Reallocating Accelerometer-Assessed Sedentary Time to Light or Moderate- to Vigorous-Intensity Physical Activity Reduces Frailty Levels in Older Adults: An Isotemporal Substitution Approach in the TSHA Study. J Am Med Dir Assoc. 2018;19(2):185 e1-6.

6. Sun F, Norman IJ, While AE. Physical activity in older people: a systematic review. BMC public health. 2013;13:449

7. Matthews CE, Chen KY, Freedson PS, Buchowski MS, Beech BM, Pate RR, et al. Amount of time spent in sedentary behaviors in the United States, 2003-2004. Am J Epidemiol. 2008;167(7):875-81.

8. Del Pozo-Cruz B, Manas A, Martin-Garcia M, Marin-Puyalto J, Garcia-Garcia FJ, Rodriguez-Manas L, et al. Frailty is associated with objectively assessed sedentary behaviour patterns in older adults: evidence from the Toledo study for healthy aging (TSHA). PLoS One. 2017;12(9):e0183911.

9. Kehler DS, Clara I, Hiebert B, Stammers AN, Hay JL, Schultz A, et al. The association between bouts of moderate to vigorous physical activity and patterns of sedentary behavior with frailty. Exp Gerontol. 2018;104:28-34.

10. Manas A, Del Pozo-Cruz B, Garcia-Garcia FJ, Guadalupe-Grau A, Ara I. Role of objectively measured sedentary behaviour in physical performance, frailty and mortality among older adults: a short systematic review. Eur J Sport Sci. 2017;17(7):940-53.

11. Bakrania K, Edwardson CL, Bodicoat DH, Esliger DW, Gill JM, Kazi A, et al. Associations of mutually exclusive categories of physical activity and sedentary time with markers of cardiometabolic health in English adults: a cross-sectional analysis of the health survey for England. BMC Public Health. 2016:16:25

12. Loprinzi PD, Lee $H$, Cardinal BJ. Daily movement patterns and biological markers among adults in the United States. Prev Med. 2014;60:128-30.

13. Bayán-Bravo A, Pérez-Tasigchana RF, López-García E, Martínez-Gómez D, Rodríguez-Artalejo F, Guallar-Castillón P. The association of major patterns of physical activity, sedentary behavior and sleeping with mortality in older adults. J Sports Sci. 2018:1-10.

14. Garcia-Garcia FJ, Gutierrez Avila G, Alfaro-Acha A, Amor Andres MS, Lanza M DLADLT, Escribano Aparicio MV, et al. The prevalence of frailty syndrome in an older population from Spain. The Toledo study for healthy aging. J Nutr Health Aging. 2011;15(10):852-6.

15. Manas A, Pozo-Cruz BD, Rodriguez-Gomez I, Losa-Reyna J, RodriguezManas L, Garcia-Garcia FJ, et al. Can physical activity offset the detrimental consequences of sedentary time on frailty? A moderation analysis in 749 older adults measured with accelerometers. J Am Med Dir Assoc. 2019.

16. Garcia-Garcia FJ, Carcaillon L, Fernandez-Tresguerres J, Alfaro A, Larrion JL, Castillo C, et al. A new operational definition of frailty: the Frailty Trait Scale. J Am Med Dir Assoc. 2014;15(5):371.e7-e13.

17. Schuit AJ, Schouten EG, Westerterp KR, Saris WH. Validity of the physical activity scale for the elderly (PASE): according to energy expenditure assessed by the doubly labeled water method. J Clin Epidemiol. 1997;50(5):541-6. 
18. del Ser Quijano T, Sanchez Sanchez F, Garcia de Yebenes MJ, Otero Puime A, Zunzunegui MV, Munoz DG. [Spanish version of the 7 Minute screening neurocognitive battery. Normative data of an elderly population sample over 70]. Neurologia (Barcelona, Spain). 2004;19(7):344-358.

19. Guralnik JM, Simonsick EM, Ferrucci L, Glynn RJ, Berkman LF, Blazer DG, et al. A short physical performance battery assessing lower extremity function: association with self-reported disability and prediction of mortality and nursing home admission. J Gerontol. 1994;49(2):M85-94.

20. Fowkes FG, Low LP, Tuta S, Kozak J. Ankle-brachial index and extent of atherothrombosis in 8891 patients with or at risk of vascular disease: results of the international AGATHA study. Eur Heart J. 2006;27(15):1861-7.

21. Jones CJ, Rikli RE, Beam WC. A 30-s chair-stand test as a measure of lower body strength in community-residing older adults. Res Q Exerc Sport. 1999;70(2):113-9.

22. Guralnik JM, Ferrucci L, Pieper CF, Leveille SG, Markides KS, Ostir GV, et al. Lower extremity function and subsequent disability: consistency across studies, predictive models, and value of gait speed alone compared with the short physical performance battery. J Gerontol A Biol Sci Med Sci. 2000;55(4):M221-31.

23. Penninx BW, Ferrucci L, Leveille SG, Rantanen T, Pahor M, Guralnik JM. Lower extremity performance in nondisabled older persons as a predictor of subsequent hospitalization. J Gerontol A Biol Sci Med Sci. 2000;55(11):M691-7.

24. Cesari M, Onder G, Zamboni V, Manini T, Shorr Rl, Russo A, et al. Physical function and self-rated health status as predictors of mortality: results from longitudinal analysis in the ilSIRENTE study. BMC Geriatr. 2008;8:34.

25. Rodríguez-Gómez I, Mañas A, Losa-Reyna J, Rodríguez-Mañas L, Chastin S, Alegre $L$, et al. Associations between sedentary time, physical activity and bone health among older people using compositional data analysis. PloS one. 2018;13(10):e0206013-e.

26. Chudyk AM, McAllister MM, Cheung HK, McKay HA, Ashe MC. Are we missing the sitting? Agreement between accelerometer non-wear time validation methods used with older adults' data. Cogent Med. 2017:4: 1313505.

27. Rodríguez-Gómez I, Mañas A, Losa-Reyna J, Rodríguez-Mañas L, Chastin SF, Alegre LM, et al. The impact of movement behaviors on bone health in elderly with adequate nutritional status: compositional data analysis depending on the frailty status. Nutrients. 2019;11(3):582.

28. Freedson PS, Melanson E, Sirard J. Calibration of the computer science and applications, Inc. accelerometer. Med Sci Sports Exerc. 1998;30(5):777-81.

29. Kozakova M, Palombo C, Morizzo C, Nolan JJ, Konrad T, Balkau B, et al. Effect of sedentary behaviour and vigorous physical activity on segment-specific carotid wall thickness and its progression in a healthy population. Eur Heart J. 2010;31(12):1511-9.

30. World Health Organization. Global Recommendations on Physical Activity for Health. WHO Press. Geneva: World Health Organization; 2010.

31. Hamilton MT, Healy GN, Dunstan DW, Zderic TW, Owen N. Too little exercise and too much sitting: inactivity physiology and the need for new recommendations on sedentary behavior. Current cardiovascular risk reports. 2008;2(4):292-8.

32. Chastin SFM, Palarea-Albaladejo J, Dontje ML, Skelton DA. Combined effects of time spent in physical activity, sedentary behaviors and sleep on obesity and cardio-metabolic health markers: a novel compositional data analysis approach. PLoS One. 2015;10(10):e0139984.

33. Fried LP. Interventions for Human Frailty: Physical Activity as a Model. Cold Spring Harb Perspect Med. 2016;6(6).

34. Santos DA, Silva AM, Baptista F, Santos R, Vale S, Mota J, et al. Sedentary behavior and physical activity are independently related to functional fitness in older adults. Exp Gerontol. 2012:47(12):908-12.

35. Trudelle-Jackson E, Jackson AW. Do Older Adults Who Meet 2008 Physical Activity Guidelines Have Better Physical Performance Than Those Who Do Not Meet? J Geriatr Phys Ther (2001). 2018;41(3):180-185.

36. Ekelund U, Steene-Johannessen J, Brown WJ, Fagerland MW, Owen N, Powell KE, et al. Does physical activity attenuate, or even eliminate, the detrimental association of sitting time with mortality? A harmonised metaanalysis of data from more than 1 million men and women. Lancet (London, England). 2016;388(10051):1302-1310.

37. Pollock RD, Duggal NA, Lazarus NR, Lord JM, Harridge SDR. Cardiorespiratory fitness not sedentary time or physical activity is associated with cardiometabolic risk in active older adults. Scand J Med Sci Sports. 2018; 28(6):1653-60
38. Dunstan DW, Kingwell BA, Larsen R, Healy GN, Cerin E, Hamilton MT, et al. Breaking up prolonged sitting reduces postprandial glucose and insulin responses. Diabetes Care. 2012;35(5):976-83.

39. McCarthy M, Edwardson CL, Davies MJ, Henson J, Bodicoat DH, Khunti K, et al. Fitness moderates glycemic responses to sitting and light activity breaks. Med Sci Sports Exerc. 2017;49(11):2216-22

40. Buman MP, Hekler EB, Haskell WL, Pruitt L, Conway TL, Cain KL, et al. Objective light-intensity physical activity associations with rated health in older adults. Am J Epidemiol. 2010;172(10):1155-65.

41. Hamer M, de Oliveira C, Demakakos P. Non-exercise physical activity and survival: English longitudinal study of ageing. Am J Prev Med. 2014;47(4):452-60

42. Chastin SFM, De Craemer M, De Cocker K, Powell L, Van Cauwenberg J, Dall $P$, et al. How does light-intensity physical activity associate with adult cardiometabolic health and mortality? Systematic review with meta-analysis of experimental and observational studies. Br J Sports Med. 2018.

43. Wolff-Hughes DL, Fitzhugh EC, Bassett DR, Churilla JR. Total activity counts and Bouted minutes of moderate-to-vigorous physical activity: relationships with Cardiometabolic biomarkers using 2003-2006 NHANES. J Phys Act Health. 2015:12(5):694-700.

44. Pavasini R, Guralnik J, Brown JC, di Bari M, Cesari M, Landi F, et al. Short physical performance battery and all-cause mortality: systematic review and meta-analysis. BMC Med. 2016;14:215

45. Migueles JH, Cadenas-Sanchez C, Ekelund U, Delisle Nystrom C, MoraGonzalez J, Lof M, et al. Accelerometer Data Collection and Processing Criteria to Assess Physical Activity and Other Outcomes: A Systematic Review and Practical Considerations. Sports medicine (Auckland, NZ). 2017.

46. An HS, Kim Y, Lee JM. Accuracy of inclinometer functions of the activPAL and ActiGraph GT3X+: a focus on physical activity. Gait \& posture. 2017:51:174-80.

47. Judice PB, Santos DA, Hamilton MT, Sardinha LB, Silva AM. Validity of GT3X and Actiheart to estimate sedentary time and breaks using ActivPAL as the reference in free-living conditions. Gait \& posture. 2015:41(4):917-22.

\section{Publisher's Note}

Springer Nature remains neutral with regard to jurisdictional claims in published maps and institutional affiliations.

Ready to submit your research? Choose BMC and benefit from:

- fast, convenient online submission

- thorough peer review by experienced researchers in your field

- rapid publication on acceptance

- support for research data, including large and complex data types

- gold Open Access which fosters wider collaboration and increased citations

- maximum visibility for your research: over $100 \mathrm{M}$ website views per year

At $\mathrm{BMC}$, research is always in progress.

Learn more biomedcentral.com/submissions 